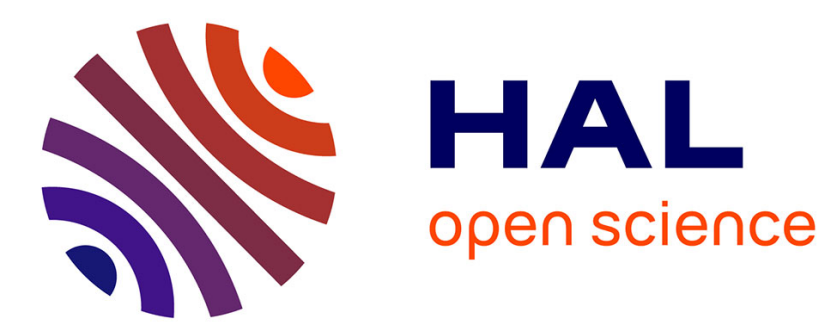

\title{
Waiting time distributions for clusters of IP3 receptors
}

Erin R. Higgins, Heiko Schmidle, Martin Falcke

\section{To cite this version:}

Erin R. Higgins, Heiko Schmidle, Martin Falcke. Waiting time distributions for clusters of IP3 receptors. Journal of Theoretical Biology, 2009, 259 (2), pp.338. 10.1016/j.jtbi.2009.03.018 . hal00554593

\section{HAL Id: hal-00554593 \\ https://hal.science/hal-00554593}

Submitted on 11 Jan 2011

HAL is a multi-disciplinary open access archive for the deposit and dissemination of scientific research documents, whether they are published or not. The documents may come from teaching and research institutions in France or abroad, or from public or private research centers.
L'archive ouverte pluridisciplinaire HAL, est destinée au dépôt et à la diffusion de documents scientifiques de niveau recherche, publiés ou non, émanant des établissements d'enseignement et de recherche français ou étrangers, des laboratoires publics ou privés. 


\section{Author's Accepted Manuscript}

Waiting time distributions for clusters of IP3 receptors

Erin R. Higgins, Heiko Schmidle, Martin Falcke

PII: $\quad$ S0022-5193(09)00129-5

DOI: $\quad$ doi:10.1016/j.jtbi.2009.03.018

Reference: $\quad$ YJTBI 5505

To appear in: $\quad$ Journal of Theoretical Biology

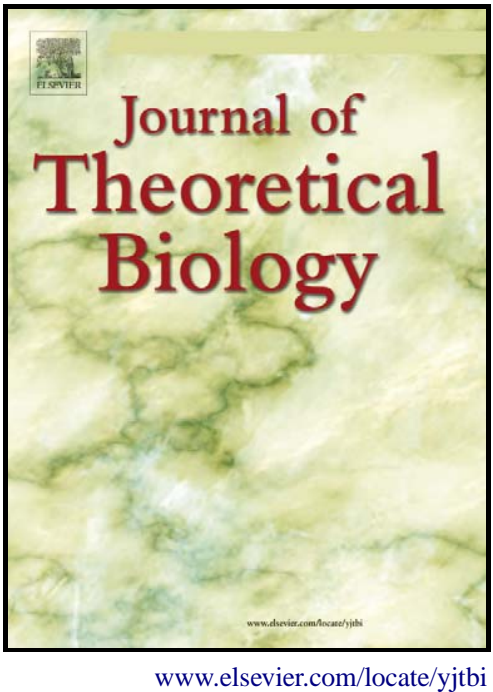

Received date: 10 December 2008

Revised date: 2 February 2009

Accepted date: $\quad 11$ March 2009

Cite this article as: Erin R. Higgins, Heiko Schmidle and Martin Falcke, Waiting time distributions for clusters of IP3 receptors, Journal of Theoretical Biology (2009), doi:10.1016/j.jtbi.2009.03.018

This is a PDF file of an unedited manuscript that has been accepted for publication. As a service to our customers we are providing this early version of the manuscript. The manuscript will undergo copyediting, typesetting, and review of the resulting galley proof before it is published in its final citable form. Please note that during the production process errors may be discovered which could affect the content, and all legal disclaimers that apply to the journal pertain. 


\title{
Waiting Time Distributions For Clusters of $\mathrm{IP}_{3}$ Receptors
}

\author{
Erin R. Higgins ${ }^{\mathrm{a}}$, Heiko Schmidle ${ }^{\mathrm{b}}$ and Martin Falcke $\mathrm{S}^{\mathrm{a}, \mathrm{b}, *}$ \\ ${ }^{a}$ Helmholtz Centre for Materials and Energy, Glienicker Straße, 14109 Berlin, \\ Germany \\ ${ }^{\mathrm{b}}$ Max Delbrück Centre for Molecular Medicine, Robert Rössle Strasse 10, 13125 \\ Berlin-Buch, Germany \\ *Corresponding author: Tel: +49 (0)30 8062 x2627, Fax: +49 (0)30 8062 2098, \\ email: falcke@hmi.de
}

\begin{abstract}
Inositol 1, 4,5-trisphosphate $\left(\mathrm{IP}_{3} \mathrm{R}\right)$ receptors control release of $\mathrm{Ca}^{2+}$ from the endoplasmic reticulum into the cytosol of a cell. The binding of both 1,4,5trisphosphate $\left(\mathrm{IP}_{3}\right)$ and activating $\mathrm{Ca}^{2+}$ is required for the receptor to open. At high $\mathrm{Ca}^{2+}$ concentrations, $\mathrm{IP}_{3} \mathrm{Rs}$ are inhibited. $\mathrm{IP}_{3} \mathrm{Rs}$ are composed of four identical subunits and form in clusters. Many models have been proposed to describe how the binding of $\mathrm{IP}_{3}$ and $\mathrm{Ca}^{2+}$ to subunits results in the opening and closing of $\mathrm{IP}_{3}$ Rs. Here we compare the opening and closing probability distributions for clusters of $\mathrm{IP}_{3} \mathrm{Rs}$, resulting from three different models. The distributions are calculated both analytically, using a method we have developed, and with simulations. We found significant differences in the behaviour of the three models as the $\mathrm{Ca}^{2+}$ and $\mathrm{IP}_{3}$ concentrations are varied.
\end{abstract}

Running title: Waiting Time Distributions

Keywords: Inositol 1,4,5-trisphosphate receptor, calcium dynamics, ion channels 


\section{Introduction}

The inositol 1,4,5-trisphosphate receptor $\left(\mathrm{IP}_{3} \mathrm{R}\right)$ acts as a channel which controls release of $\mathrm{Ca}^{2+}$ from the endoplasmic reticulum (ER) into the cytosol of a cell. The $\mathrm{IP}_{3} \mathrm{Rs}$ release $\mathrm{Ca}^{2+}$ in response to an increase in the concentration of inositol 1,4,5trisphosphate $\left(\mathrm{IP}_{3}\right)$ in the cytosol, which results from a stimulus, such as the binding of a hormone to the cell membrane. $\mathrm{IP}_{3}$ Rs open and close randomly, and the open and closing probabilities are determined by the binding of $\mathrm{IP}_{3}$ and $\mathrm{Ca}^{2+}$. A small increase in the concentration of cytosolic $\mathrm{Ca}^{2+}$ stimulates channel activation, whereas a large increase causes inhibition of the $\mathrm{IP}_{3} \mathrm{R}$. This results in a bell-shaped dependence of the open probability on the cytosolic $\mathrm{Ca}^{2+}$ concentration. Activation of the channels takes place on a faster time scale than inhibition. $\mathrm{IP}_{3} \mathrm{Rs}$ are found in clusters, and so the release of $\mathrm{Ca}^{2+}$ resulting from the opening of one channel in a cluster, stimulates the opening of other channels in the cluster. The release of $\mathrm{Ca}^{2+}$ from the cluster is referred to as a $\mathrm{Ca}^{2+}$ puff. Puffs are the elemental events of intracellular $\mathrm{Ca}^{2+}$ release.

Various models have been proposed for describing the $\operatorname{IP}_{3} \mathrm{R}[15,7]$. Here we have compared the opening and closing probability distributions resulting from three different models. These include the model proposed in Rüdiger et al. and Shuai et al. $[10,12]$ (model 1), the same model modified to include sequential binding of $\mathrm{IP}_{3}$ and $\mathrm{Ca}^{2+}$ as suggested by Adkins and Taylor [1] (model 2), and the model developed by Sneyd and Dufour [14] (model 3) as given in reference Ullah et al. [19]. The $\mathrm{IP}_{3} \mathrm{R}$ is comprised of four subunits, which are assumed to be identical, and these three models each describe the transitions between subunit states, most of which result from the binding and release of $\mathrm{IP}_{3} \mathrm{R}$ and $\mathrm{Ca}^{2+}$. Model 1 [12] is closely based on the DeYoung-Keizer model [3], and assumes that a subunit has binding sites for $\mathrm{IP}_{3}$, activating $\mathrm{Ca}^{2+}$ and inhibitory $\mathrm{Ca}^{2+}$. The model by Sneyd and Dufour $[19,14,7]$ was designed to agree with several experimental observations.

While it has been known for about a decade now that puffs are random release events, we recently showed that spike sequences of intracellular $\mathrm{Ca}^{2+}$ oscillations are random events also [13]. The random opening and closing of individual channels, i.e. 
the random state transitions of their subunits, is believed to be the most important source of noise causing the random behavior of the whole system [6]. That requires stochastic theory across all structural levels from a channel subunit up to cell level for modelling. However, existing subunit models typically comprise 6 or more subunit states, leading to hundreds of channel states and many thousands of channel cluster states. That makes stochastic theory on cluster level already almost impossible, not to mention cell level. Thus theoretical studies on puff characteristics used either extremely simplified channel models (with two subunit states [11], two or four channel states [9]) or relied on simulations [19].

Recently we presented a method which circumvents writing down the master equation of the channel molecule with its huge number of states and gets by with the subunit master equation only in calculations of waiting time distributions for channel clusters [18]. Here we follow a different approach. Channel states are defined by the number of subunits in subunit states. We derive the channel Master equation from the subunit Master equation. Subsequently, we lump channel states into aggregates based on time scale separation. Standard methods for calculating cluster properties can then be applied to the Master equation defined for the lumped states.

Using these approximations, we compared the probability distributions for the first opening time of a cluster and the first closing time of a cluster, at various $\mathrm{IP}_{3} \mathrm{R}$ and $\mathrm{Ca}^{2+}$ concentrations. These distributions were calculated analytically as well as through simulations, and assume a cluster consists of five channels. The three models exhibit significantly different behaviour. The sequential binding of $\mathrm{IP}_{3}$ and $\mathrm{Ca}^{2+}$ imposed in model 2 results in longer expected opening times compared to model 1 and longer expected closing times compared to both models 1 and 3. Model 3 has a shorter expected closing time than models 1 and 2, but has a much longer expected opening time at low $\mathrm{IP}_{3}$ concentrations and high $\mathrm{Ca}^{2+}$ concentrations. These probability distributions are calculated assuming the $\mathrm{Ca}^{2+}$ concentration in the vicinity of the cluster remains constant. That is, we use one fixed value for the $\mathrm{Ca}^{2+}$ concentration when the cluster is closed, and another fixed value for $\mathrm{Ca}^{2+}$ when the cluster is open, i.e., when at least one channel is open. We have also looked at the effect of using a 
variable $\mathrm{Ca}^{2+}$ concentration while the cluster is open, whereby a closed channel sees a lower concentration than an open channel. The resulting probability distributions are very similar to when a fixed value is used, in all three models.

\section{Single Channel Activation}

We would like to calculate $F(A, t \mid I, 0)$, the probability distribution for a channel first being activated at time $t$, given it is in state $I$ at time $t=0$. $A$ denotes the set of active channel states, and we say a channel is activated when it is in one of these states. We are assuming we have the subunit transition matrix $W$, where $w_{i, j} d t$ gives the probability for a subunit to move from state $x_{j}$ to state $x_{i}$ in infinitesimal time $d t$, for $i \neq j . w_{j, j}=-\sum_{i=1, i \neq j}^{n} w_{i, j}$ where $n$ is the number of subunit states. The probability for a subunit to stay in state $x_{i}$ during time $d t$ is $1+w_{i, i} d t$.

We demonstrate how to build the subunit transition matrix $W$ with model 1 (see section 5 for a detailed description of the model). First, we order the subunit states: $(110+$ Active, 111, 010, 011, 100, 101,000,001). For instance the transition rate from state 100 to $110+$ Active is $a_{5} c$ where $c$ denotes the $\mathrm{Ca}^{2+}$ concentration. Now we can build the $8 \times 8$ subunit transition matrix

$$
W=\left(\begin{array}{cccccccc}
-\sum_{1} & b_{2} & a_{1} \cdot p & 0 & a_{5} \cdot c & 0 & 0 & 0 \\
\frac{b_{0} a_{2}}{a_{0}+b_{0}} \cdot c & -\sum_{2} & 0 & a_{3} \cdot p & 0 & a_{5} \cdot c & 0 & 0 \\
\frac{b_{0} b_{1}}{a_{0}+b_{0}} & 0 & -\sum_{3} & b_{4} & 0 & 0 & a_{5} \cdot c & 0 \\
0 & b_{3} & a_{4} \cdot c & -\sum_{4} & 0 & 0 & 0 & a_{5} \cdot c \\
\frac{b_{0} b_{5}}{a_{0}+b_{0}} & 0 & 0 & 0 & -\sum_{5} & b_{2} & a_{1} \cdot p & 0 \\
0 & b_{5} & 0 & 0 & a_{2} \cdot c & -\sum_{6} & 0 & a_{3} \cdot p \\
0 & 0 & b_{5} & 0 & b_{1} & 0 & -\sum_{7} & b_{4} \\
0 & 0 & 0 & b_{5} & 0 & b_{3} & a_{4} \cdot c & -\sum_{8}
\end{array}\right) .
$$

All entries have dimension $s^{-1}, p$ denotes the $\mathrm{IP}_{3}$ concentration. $-\sum_{i}$ represents the negative sum of the $i$ th column.

We can use $W$ to form the channel state transition matrix $P$. An entry in $P, p_{i, j} d t$, gives the probability for a channel to move from state $X_{j}$ to state $X_{i}$ in infinitesimal 
time $d t$, for $i \neq j$. The diagonal elements are given by $p_{j, j}=-\sum_{i=1, i \neq j}^{r} p_{i, j}$. The off-diagonal elements of $P$ are found as follows. A channel state is represented by a vector $V$ where an element of the vector, $v_{i}$, gives the number of subunits in subunit state $x_{i}$. Thus the length of $V$ is $n$, and the sum of the elements in $V$ is the number of subunits in each channel, $h$. The transition probability $p_{i, j}$ is 0 , if $X_{j}$ and $X_{i}$ differ by more than one subunit and $p_{i, j}=\phi w_{k, l}$, if $X_{i}$ can be reached from $X_{j}$ by one subunit in state $x_{l}$ moving to state $x_{k}$, and $X_{j}$ has $\phi$ subunits in state $x_{l}$.

To find $F(A, t \mid I, 0)$ we first remove all rows and columns in $P$ which correspond to active channel states and denote the new matrix by $\bar{P}$. That is, if $X_{i} \in A$ then we remove row $i$ and column $i$. This means if the system reaches an active state, it cannot leave it. Let $y\left(X_{i}, t \mid I, 0\right)$ be the probability of being in nonactive state $X_{i}$ at time $t$ and let $y=\left\{y\left(X_{i}, t \mid I, 0\right), X_{i} \notin A\right\}$. Then $y$ can be found by solving the master equation

$$
\dot{y}=\bar{P} y
$$

Let $f(A, t \mid I, 0)$ be the probability that an activated state is reached in the time interval $[0, t]$, let $r$ be the total number of channel states and let $r_{a}$ be the number of open channel states. Then

$$
f(A, t \mid I, 0)=1-\sum_{i: X_{i} \notin A} y\left(X_{i}, t \mid I, 0\right)=1-\sum_{i=1}^{r-r_{a}} \sum_{k=1}^{r-r_{a}} c_{k} V_{k, i} e^{\lambda_{k} t}
$$

where $\lambda_{k}$ and $V_{k}, k=1 \ldots r-r_{a}$ are the eigenvalues and eigenvectors respectively, of $\bar{P}$ and where $c_{k}, k=1 \ldots r-r_{a}$ are determined by the initial conditions. Then

$$
F(A, t \mid I, 0)=\frac{d f(A, t \mid I, 0)}{d t}=-\sum_{i=1}^{r-r_{a}} \sum_{k=1}^{r-r_{a}} c_{k} \lambda_{k} V_{k, i} e^{\lambda_{k} t}
$$

\section{Cluster Activation}

A cluster is first activated when any of the channels in the cluster are first activated. We assume the initial state of a channel is unknown, so we use the weighted average over all possible initial channel states, where the weights are given by the probabilities of each channel state occurring at rest. Let $F_{c h}(A, t)$ denote the probability distribution for a channel to first open at time $t$ given that the initial state is unknown 
and let $F_{\text {open }}(A, t)$ denote the probability distribution for a cluster of channels to first open at time $t$ (opening time distribution). Then $F_{\text {open }}(A, t)$, for $t>0$, is given by

$$
F_{\text {open }}(A, t)=N F_{c h}(A, t) G(A, t)^{N-1}
$$

where

$$
G(A, t)=1-\int_{0}^{t} F_{c h}(A, \tau) d \tau
$$

gives the probability that a channel has not been activated by time $t$ and $N$ is the number of channels in the cluster. That is, the probability for the cluster to be first activated in the time interval $[t, t+d t]$ is given by the probability that one of the $N$ channels is first activated in the time interval $[t, t+d t]$ and all other channels have not yet been activated.

When $t=0$, if we used the method above to calculate $F_{\text {open }}(A, 0)$, this would mean that the probability for the cluster to open in the interval $[0, d t]$ is given by the probability that one of the $N$ channels opens in $[0, d t]$, while the other $N-1$ do not. However, this is not correct. The probability that the cluster opens in $[0, d t]$ is given by the probability that at least one of the $N$ channels opens in $[0, d t]$, while the other $N-1$ channels can either open or not open. $F_{\text {open }}(A, 0)$ can be calculated using

$$
F_{\text {open }}(A, 0)=1-\left(1-F_{c h}(A, 0)\right)^{N} \text {. }
$$

That is, the probability that the cluster opens instantly is the negation of no channel opening instantly.

We use the non-open states weighted according to their resting probabilities as initial condition for calculating $F(A, t)$ for $t>0$. The resting probabilities for the initial channel states are given by the normalized eigenvector of $P$ corresponding to the eigenvalue $\lambda=0$.

\section{Cluster Closure}

The cluster of channels is considered closed when all channels in the cluster are closed. Let $F_{\text {close }}(A, t)$ denote the probability distribution for a cluster of channels 
to first close at time $t$ if it has opened at time $\mathrm{t}=0$ (closing time distribution). To calculate $F_{\text {close }}(A, t)$, we find the cluster state transition matrix, $M$, from the channel state transition matrix $P$, in an analogous way to how we found $P$ from the subunit state transition matrix, $W$. That is, a cluster state is represented by a vector with length given by the number of channel states. An entry in the vector gives the number of channels in the corresponding channel state. Thus, the sum of the elements in the vector is the number of channels in the cluster, $N$. The probability of a transition between cluster states occurring which involves more than one channel changing state instantaneously is infinitely small compared to the probability of a transition where only one channel changes state, and so for such transitions, the entry in $M$ is 0 . If cluster state $\mathcal{X}_{i}$ can be reached from state $\mathcal{X}_{j}$ by one channel in state $X_{l}$ moving to state $X_{k}$, and $\mathcal{X}_{j}$ has $\phi$ channels in state $X_{l}$, then the entry in $M, m_{i, j}=\phi p_{k, l}$. The diagonal entries of $M$ are such that the columns of $M$ sum to 0.

Once $M$ has been constructed, $F_{\text {close }}(A, t)$ can be found analogously to the way in which $F(A, t \mid I, 0)$ is found in section 2 . That is, let $\bar{M}$ be the cluster state transition matrix with rows and columns corresponding to closed cluster states removed. Let $\rho$ be the total number of cluster states and let $\rho_{a}$ be the number of closed cluster states. Let $\mu_{k}$ and $U_{k}, k=1 \ldots \rho-\rho_{a}$ be the eigenvalues and eigenvectors respectively, of $\bar{M}$. Then

$$
F_{\text {close }}(A, t)=-\sum_{i=1}^{\rho-\rho_{a}} \sum_{k=1}^{\rho-\rho_{a}} d_{k} \mu_{k} U_{k, i} e^{\mu_{k} t}
$$

where $d_{k}, k=1 \ldots \rho-\rho_{a}$ are determined by the initial conditions.

We choose as the initial probabilities for a channel to be in each of the channel states the probabilities at the expected cluster opening time. That is, we begin with the resting probabilities, then solve the master equation,

$$
\dot{z}(t)=P_{0} z(t)
$$

where $z(t)$ gives the probabilities of being in the channel states at time $t$, and $P_{0}$ is found from $P$ by setting transition rates out of open states to 0 , because we ave interested in those trajectories only, which did not go through open states. $z(t)$ is then evaluated at the expected opening time for the cluster, which is calculated from 
$F_{\text {open }}(A, t)$. Initially, we assume one channel in the cluster is open, while the others are closed. To enforce this condition, we find from $z(t)$ the conditional probability of being in each state where exactly one channel is open, given that the cluster is in one such state. All other cluster states have an initial probability of zero.

The large number of cluster states presents a problem when calculating $F_{\text {close }}(A, t)$. The number of channel states, $r$ is given by $r=\left(\begin{array}{c}h+n-1 \\ n-1\end{array}\right)$. Recall, $n$ is the number of subunit states and $h$ is the number of subunits per channel. The number of cluster states, $\rho$, is given by $\rho=\left(\begin{array}{c}N+r-1 \\ r-1\end{array}\right)$. Recall, $N$ is the number of channels in the cluster. So $\rho$ is very large for the values of $n, h$ and $N$ we require, which results in the above method for finding $F_{\text {close }}(A, t)$ being too slow and requiring too much memory to be feasible.

To overcome the problem of there being too many cluster states, we reduce the number of channel states before finding $M$. We developed a reduction method motivated by and similar to that described in Huisinga et al. [4]. It is based on the theory of weakly coupled Markov chains. The weakly coupled state aggregates are identified by a Perron cluster analysis in the study by Huisinga et al. We replace cluster identification by a simpler method. In one case we compared our results with those of the method used in [4] and found them to be identical.

We identify aggregates by repeatedly finding the largest transition rate in the channel state transition matrix $P$, then combining the two states connected by the transition, into one aggregate state. In general we combine two states $X_{i}, X_{j}$ to an aggregate state $\left\{X_{i}, X_{j}\right\}$ and after repeating the reduction process we get a set of aggregate states of the form $\left\{X_{a_{i}}, i=1 \ldots m\right\}$. The transition rate for moving from the aggregate state $\left\{X_{a_{i}}, i=1 \ldots m_{1}\right\}$ to the aggregate state $\left\{X_{b_{i}}, i=1 \ldots m_{2}\right\}$ is then given by

$$
\sum_{j=1}^{m_{2}}\left[\left(\sum_{i=1}^{m_{1}} p_{b_{j}, a_{i}} V_{0}\left(a_{i}\right)\right) / \sum_{i=1}^{m_{1}} V_{0}\left(a_{i}\right)\right] .
$$

$V_{0}$ represents the stationary solution of the whole un-reduced system and $p_{b_{j}, a_{i}}$ denotes the transition probability from state $a_{i}$ to $b_{j}$. This is repeated until the number of aggregate channel states is sufficiently small that the number of cluster states is small 
enough for computations to be feasible or till the stop criterion has been reached (see section 7).

This process of combining channel states results in open and closed channel states being combined. However, as we require open states to be distinct from closed states, we divide the aggregate states which contain both open and closed states into two separate states, one containing only the closed states and one containing only the open states. After these aggregate states are split, transition rates to and from the new aggregate states must be found.

\section{Subunit Models}

We have compared results using three models of $\mathrm{IP}_{3}$ channel subunits. These include the nine state model of Rüdiger et al. [10] which we have reduced to eight states, the same model but with conditions of sequential binding of $\mathrm{Ca}^{2+}$ and $\mathrm{IP}_{3}$ imposed, and the model of Sneyd and Dufour [14] modified and reduced to six states as given by Ullah and Jung [19].

\subsection{Model 1}

Model 1 is based on the DeYoung-Keizer model [3], whereby the subunit has an $\mathrm{IP}_{3}$ binding site, an activating $\mathrm{Ca}^{2+}$ binding site and an inhibitory $\mathrm{Ca}^{2+}$ binding site. Subunit states are represented by the triplet $(i j k)$, where $i, j$ and $k$ represent these three binding sites respectively. An occupied site is represented with a 1 and an unoccupied site with a 0 . In the DeYoung-Keizer model [3], the subunit is considered active when it is in state (110). That is, when the $\mathrm{IP}_{3}$ and activating $\mathrm{Ca}^{2+}$ binding sites are occupied but the inhibitory $\mathrm{Ca}^{2+}$ binding site is not occupied. Rüdiger et al. [10] have added an extra state labelled 'Active' with transitions to and from state (110). The subunit is only active when it is in this state. This model is illustrated in Fig. 1. We have combined the 'Active' state and state (110) into one state by assuming the transition rates between the two states are fast, and so reduced the model to eight states. We have used the transition rates given by Shuai et al. [12], 
which are listed in the Appendix.

A channel contains four such subunits, and is considered open when any three of the four subunits are in the active state.

\section{$5.2 \quad$ Model 2}

It had been suggested by Adkins and Taylor [1] that the binding of $\mathrm{Ca}^{2+}$ to the activating and inhibitory binding sites may be affected by whether or not the $\mathrm{IP}_{3}$ binding site is occupied. Specifically, if $\mathrm{IP}_{3}$ is bound, then $\mathrm{Ca}^{2+}$ cannot bind to the

inhibitory $\mathrm{Ca}^{2+}$ binding site, and if $\mathrm{IP}_{3}$ is not bound, then $\mathrm{Ca}^{2+}$ cannot bind to the activating $\mathrm{Ca}^{2+}$ binding site. Thus, model 2 uses the same scheme as model 1, but the activating $\mathrm{Ca}^{2+}$ binding rate when $\mathrm{IP}_{3}$ is not bound, and the inhibitory $\mathrm{Ca}^{2+}$ binding rate when $\mathrm{IP}_{3}$ is bound, are made small (they are set to $1.0 \times 10^{-3} \mu \mathrm{M}^{-1} \mathrm{~s}^{-1}$ ). In order to maintain the condition of detailed balance, the corresponding reverse transition rates (the $b_{i}$ values) have also been made small, so that the dissociation constants, $K_{i}=b_{i} / a_{i}$ remain the same. The transitions that are made slow are indicated by dotted lines in Fig. 1.

\section{$5.3 \quad$ Model 3}

Sneyd and Dufour [14] describe a 10 state model of the $\mathrm{IP}_{3} \mathrm{R}$ subunit. It includes one $\mathrm{IP}_{3}$ binding site, two binding sites for $\mathrm{Ca}^{2+}$ activation and one binding site for $\mathrm{Ca}^{2+}$ inactivation. The Sneyd and Dufour [14] model was designed to produce agreement with a number of results from experimental data. Falcke [7] modified the model to overcome a problem involving a lack of $\mathrm{Ca}^{2+}$ conservation in triangular motifs employed to achieve saturating binding kinetics. These triangular motifs are replaced by square motifs Fig. 2 B, and the full model contains then thirteen states. The resulting transition diagram is shown in Fig. 2. We have used the parameter values given by Ullah and Jung [19], but changed them slightly in order to obey detailed balance. They can be found in the Appendix.

A channel consists of four of these subunits, and is considered open when all four 
are in state $O^{\prime}, \tilde{O}, \bar{O}$ (the open states) or state $\tilde{A}, \bar{A}$ (the activated states) or an intermediate combination, such as one subunit in state $O^{\prime}$ and three in state $\bar{A}$.

\section{Results}

We show results for a cluster consisting of five channels, using various values for the $\mathrm{Ca}^{2+}$ concentration when the cluster is closed, $c_{\text {close }}$, the $\mathrm{Ca}^{2+}$ concentration when the cluster is open, $c_{\mathrm{open}}$, and the $\mathrm{IP}_{3}$ concentration, $p$. When finding the steady channel state probabilities, we use $p=10^{-7}$ and the $\mathrm{Ca}^{2+}$ concentration $c_{\text {close }}$. When the cluster is open, we use the $\mathrm{Ca}^{2+}$ concentration $c_{\text {open }}$ and it remains fixed regardless of the number of open channels. When $c_{\text {close }}, c_{\mathrm{open}}$ and $p$ are not being varied, their values are $0.1 \mu \mathrm{M}, 100 \mu \mathrm{M}$ and $0.15 \mu \mathrm{M}$ respectively. Figures $3-9$ show the effect of changing $c_{\text {close }}, c_{\text {open }}$ and $p$ when using models 1, 2 and 3 . We present the probability distribution for the opening time $F_{\text {open }}(A, t)$ and closing time $F_{\text {close }}(A, t)$ of a cluster of channels and the expected value of the opening time distribution $T_{\text {open }}$ and closing time distribution $T_{\text {close }}$.

In Fig. $3 \mathrm{~A}, \mathrm{~B}$, we see that the opening distributions for models 1 and 2 are very similar when $c_{\text {close }}<0.1 \mu \mathrm{M}$. At large $\mathrm{Ca}^{2+}$ concentrations, model 2 has a much longer expected opening time compared to model 1 (see Fig. 4). At high $\mathrm{Ca}^{2+}$ concentrations, there is a high probability of a subunit being in state (011) at rest. In model 1, the fastest route from (011) to (110) is through the binding of $\mathrm{IP}_{3}$ followed by the release of inhibitory $\mathrm{Ca}^{2+}$. However, in model 2 this route is not fast, resulting in a longer expected opening time. When the $\mathrm{Ca}^{2+}$ concentration is low $(\sim 0.1 \mu \mathrm{M})$ subunits are more likely to initially be in state (000), from which transitions to the active state are very fast in both models. However, if the $\mathrm{Ca}^{2+}$ concentration is very low $\left(0.01 \mu \mathrm{M}\right.$ for example), the binding of $\mathrm{Ca}^{2+}$ to the activating $\mathrm{Ca}^{2+}$ site is slow, resulting in the increase in the expected opening times seen in Fig. 4 A,B.

In model 3, the single $\mathrm{Ca}^{2+}$ dependent transition rate into an active state depends on the concentration of $\mathrm{IP}_{3}$ too, which is slow for small $\mathrm{IP}_{3}$ concentration. Therefore, the effect of increasing the $\mathrm{Ca}^{2+}$ concentration is to increase the transition rates into 
the inactivated or shut states. That slows down activation (Fig. 3 C, Fig. 4) and the expected opening time does not exhibit a minimum in dependence on the $\mathrm{Ca}^{2+}$ concentration.

In Fig. $5 \mathrm{~A}-\mathrm{C}$ and Fig. $6 \mathrm{~A}, \mathrm{~B}$ we see that increasing $p$ results in an initial rapid decrease in the expected opening time. All three models exhibit high sensitivity to the $\mathrm{IP}_{3}$ concentration at low concentrations, and low sensitivity at high concentrations. Model 2 again has a long expected opening time compared to model 1 as the fastest routes to the active state in model 1 are made slow in model 2.

Fig. 7 A,B and Fig. 8 show that changing the $\mathrm{Ca}^{2+}$ concentration has a strong effect on the closing behavior of model 1 , but has very little effect on the closing time distribution of model 2. In model 2, the $\mathrm{Ca}^{2+}$ dependent transition rate out of the open state is very low, so the $\mathrm{Ca}^{2+}$ concentration has little effect on the expected closing time. The most prominent closing transition is $\mathrm{IP}_{3}$ dissociation. At high $\mathrm{Ca}^{2+}$ concentrations, model 1 closes quickly due to the binding of inhibitory $\mathrm{Ca}^{2+}$. However, in model 2 inhibitory $\mathrm{Ca}^{2+}$ cannot bind quickly until $\mathrm{IP}_{3}$ has first been released, resulting in longer expected closing times. We increased the rate of $\mathrm{IP}_{3}$ release in model 2 by a factor of 10 (that is, $K_{1}=0.036 \mu \mathrm{M}$ and $K_{3}=8.0 \mu \mathrm{M}$ ), and this decreased the expected closing time. Changing the $\mathrm{Ca}^{2+}$ concentration still has little effect on the expected closing time when the $\mathrm{IP}_{3}$ release rate is increased. Model 3 does neither exhibit any noteworthy dependence of the cluster closing rate on the $\mathrm{Ca}^{2+}$ concentration. Closing is obviously dominated by the $\mathrm{Ca}^{2+}$-independent transitions with the rate $4\left(l_{-4}+k_{-2}\right)$ (see Fig. 2). Fig. 9 A-C and Fig. 10 A,B show that increasing the $\mathrm{IP}_{3}$ concentration increases the expected closing time in all three models.

In Fig. $11 \mathrm{~A}-\mathrm{C}$ we show results using both a fixed and variable value for the $\mathrm{Ca}^{2+}$ concentration, $c_{\mathrm{open}}$. When $c_{\mathrm{open}}$ is fixed, it has a value of $100 \mu \mathrm{M}$ and applies to all channels in the cluster as soon as at least one channel is open. That corresponds to very strong spatial coupling by $\mathrm{Ca}^{2+}$ diffusion. When $c_{\text {open }}$ is variable it takes on different values depending on whether an individual channel is open or closed corresponding to weaker spatial coupling. For an open channel $c_{\text {open }}=0.1+99.9=$ 
$100 \mu \mathrm{M}$ as before. This corresponds to a base level concentration of $0.1 \mu \mathrm{M}$ and an increase of $99.9 \mu \mathrm{M}$ resulting from the opening of the cluster. For a closed channel the increase is reduced. We use $c_{\text {open }}=0.1+0.1 \times 99.9=10.09 \mu \mathrm{M}$ and $c_{\text {open }}=$ $0.1+0.01 \times 99.9=1.099 \mu \mathrm{M}$. We see that making $c_{\mathrm{open}}$ variable in this manner has very little effect.

Variable $c_{\text {open }}$ has of course no affect on the expected opening time, but could change the time course of a puff and therefore the expected closing time. Model 1 shows significant dependence of the closing time distribution on the $\mathrm{Ca}^{2+}$ concentration and so we may expect that making the $\mathrm{Ca}^{2+}$ concentration variable would have a more significant effect than seen here. A possible explanation is that channels still open fast with the smaller concentrations and channel reopening is very slow compared to channel closing. Thus if a channel closes, it is very likely that the rest of the channels will close before it has reopened, whether it sees a $\mathrm{Ca}^{2+}$ concentration of $1.099 \mu \mathrm{M}, 10.09 \mu \mathrm{M}$ or $100 \mu \mathrm{M}$.

In Fig. $12 \mathrm{~A}-\mathrm{C}$ and $13 \mathrm{~A}-\mathrm{C}$ we compare the analytic results with the simulation results, for $F_{\text {open }}(A, t) d t$ and $F_{\text {close }}(A, t) d t$ resp., using the fixed values of $c_{\text {close }}, c_{\text {open }}$ and $p$. Because our method for finding $F_{\text {close }}(A, t) d t$ relies on an approximation of the channel state transition matrix, there is potential for there to be significant errors in the analytic results. For models 1 and 2 the number of channel states is reduced from 330 to 12 and for model 3 from 1820 to 11 . Comparison of the expected values found using the analytic results and the simulation results (see Fig 8 and Fig. 10 A,B) show the agreement for model 1 and model 3 is not good for high values of $\mathrm{IP}_{3}$. Model 1 has a large expected closing time compared to the other two models which is partly due to a comparatively high open probability at large time values. Fig. 12 and 13 show that the agreement of the analytical and numerical distributions for small times is rather good. However, if the distribution is more spread out, a small error in the tail of the analytic distributions may be causing the bad agreement. 


\section{$7 \quad$ Stop criterion for Reduction}

Fig. 14 A-C show how the expected closing time for a single channel varies as the number of aggregate states is reduced. When there are 330 aggregate states for models 1 and 2 and 1820 states for model 3, the full model is being used. The calculations are done for a single channel here, as it is too computationally expensive to use more channels with a large number of aggregate states. The vertical lines mark the number of aggregate states used in the results presented in this study. Note, the horizontal axes give the number of states prior to the open states being separated out. We use 12 aggregate states for model 1, 2 and 11 for model 3 here (after separating the open states). This is the maximum number for which results could be computed due to limitations set by computational efficiency. ${ }^{1}$ We see also from Fig. 14 that this is close to the minimum value for which we can expect reasonably accurate results, when using a single channel.

It can be seen in Fig. $14 \mathrm{~A}-\mathrm{C}$ that the expected closing time changes abruptly as the number of aggregate states changes. The abrupt changes in the expected closing time correspond exactly to changes in the number of open aggregate states. There is a dramatic change for all three models and all values of $c_{\text {open }}$ when the number of open aggregates gets below a critical value. That critical value provides the stop criterion for cluster state reduction.

Fig. 15 compares exact analytic results with simulations. The error does not vary greatly as the number of channels increases from 1 to 5 , suggesting that if the results are reasonably accurate when using a single channel, they will also be reasonably accurate when using 5 channels. Thus, the method we suggest for predicting the accuracy of results after aggregation, is to compute the expected closing time for a single channel using the full model and to compare this with the expected closing time computed after the number of states has been reduced. The minimum number of aggregate states for which reasonable approximations can be expected is the number just above the dramatic change of results.

\footnotetext{
${ }^{1}$ Several hours using a UniServer 3346 with 4Dual-Core AMD Opteron 800 series CPU's
} 


\section{Discussion}

Many models describing the kinetics of $\mathrm{IP}_{3} \mathrm{R}$ subunits have been proposed. We have compared the probability distributions for the expected opening and closing times of a cluster of five $\mathrm{IP}_{3}$ Rs resulting from three different subunit models. The first model is that of Shuai et al. [12], which is similar to the DeYoung-Keizer model [3]. Here there are binding sites for $\mathrm{IP}_{3}$, activating $\mathrm{Ca}^{2+}$ and inhibitory $\mathrm{Ca}^{2+}$, resulting in eight subunit states. Shuai et al. [12] add an extra 'Active' state reachable only from the state where $\mathrm{IP}_{3}$ and activating $\mathrm{Ca}^{2+}$ are bound, and inhibitory $\mathrm{Ca}^{2+}$ is not bound. We have reduced these two states to one state by assuming the transitions between them are fast. The parameter values are such that the binding of $\mathrm{IP}_{3}$ reduces the affinity of the inhibitory $\mathrm{Ca}^{2+}$ site, and $\mathrm{Ca}^{2+}$ binding to the inhibitory site reduces the affinity of the $\mathrm{IP}_{3}$ binding site. The second model used is a modification of the first suggested by Adkins and Taylor [1]. Here the inhibitory $\mathrm{Ca}^{2+}$ binding rate when $\mathrm{IP}_{3}$ is bound is made very small, as is the activating $\mathrm{Ca}^{2+}$ binding rate when $\mathrm{IP}_{3}$ is not bound. The reverse transition rates are also made small to maintain detailed balance. The third model is a thirteen state model given by Sneyd and Dufour [14] and extended by Falcke [7] designed to accommodate various experimental findings.

We have compared the opening and closing time distributions resulting from the three models, at various $\mathrm{Ca}^{2+}$ and $\mathrm{IP}_{3}$ concentrations, as well as the expected opening and closing times. We found the three models gave significantly different results. At low $\mathrm{Ca}^{2+}$ concentrations $(<0.1 \mu \mathrm{M})$, models 1 and 2 have similar expected opening times, but at larger $\mathrm{Ca}^{2+}$ concentrations model 2 has a much longer expected opening time. When the $\mathrm{Ca}^{2+}$ concentration is large, both the activating and inhibiting $\mathrm{Ca}^{2+}$ binding sites are likely to be occupied prior to the addition of $\mathrm{IP}_{3}$. Model 1 then opens comparatively quickly due to the binding of $\mathrm{IP}_{3}$ followed by the release of inhibitory $\mathrm{Ca}^{2+}$. This route to activation is slow in model 2 as the rate for the release of inhibitory $\mathrm{Ca}^{2+}$ when $\mathrm{IP}_{3}$ is bound is small.

The models show also differences in closing behavior. Using model 1 there is a decrease in the expected closing time of a cluster, as the $\mathrm{Ca}^{2+}$ concentration increases 
from $20 \mu \mathrm{M}$ to $200 \mu \mathrm{M}$. This is due to an increased rate of $\mathrm{Ca}^{2+}$ inhibition. In model 2 the rate for $\mathrm{Ca}^{2+}$ inhibition while $\mathrm{IP}_{3}$ is bound is very slow, resulting in less sensitivity of the expected closing time to the $\mathrm{Ca}^{2+}$ concentration. Model 3 exhibits only a very weak dependence of the expected closing time on $\mathrm{Ca}^{2+}$. Using all three models, the expected cluster closing time increases monotonically with the $\mathrm{IP}_{3}$ concentration.

We have used a fixed value for the $\mathrm{Ca}^{2+}$ concentration when the cluster is open. However, the concentration around the cluster may vary depending on whether individual channels are open or closed. In Fig. 11 we use a variable $\mathrm{Ca}^{2+}$ concentration, so that channels in the cluster which are closed see a lower $\mathrm{Ca}^{2+}$ concentration than those that are open. Making the $\mathrm{Ca}^{2+}$ concentration variable has had very little impact. Fraiman et al. [8] note that the time taken for a single inactivated $\mathrm{IP}_{3} \mathrm{R}$ to become uninhibited is much longer than a typical puff duration. Our results support this statement. Consequently, the $\mathrm{Ca}^{2+}$ concentration seen by a closed $\mathrm{IP}_{3} \mathrm{R}$ has little effect, as all channels are likely to close before it has time to reopen. These results imply that puffs with these models are insensitive towards spatial arrangement of individual channels within the cluster as long as the distance is not too large. We mimicked a decay of the $\mathrm{Ca}^{2+}$ concentration on the channel distance corresponding to 3-4.6 diffusion lengths of free $\mathrm{Ca}^{2+}$.

Our method for calculating the closing time distribution of a cluster requires the number of channels in the cluster to be small $(\leq 5)$ so that the number of possible cluster states is not too large. There have been various estimates of the number of channels in a cluster. Some estimate it to be in the range of 20-30 [2, 5, 16], while Shuai et al. [12] estimate 40-70. However, Fraiman et al. [8] estimate 4 or 5, which agrees with an estimate by Swillens et al. [17] and Demuro et al. of about 10 channels in a cluster [20]. Very recent experiments suggest an average number of 4 [21].

In order to calculate the closing time distribution of a cluster, we reduced the number of channel states so that the number of cluster states was not too large. We tried different methods to reduce the system among them a rapid-equilibrium approximation: We identified the states connected by the fastest transition rates, assumed equilibrium just between these two states, lumped them accordingly and 
repeated the procedure with the new set of states. With respect to accuracy, that method was as good as the one we used here. Its only disadvantage was that it did not allow for re-separating closed and open states once the aggregates had been found.

The state aggregation introduces error to the calculation. This is particularly apparent in the poor agreement between the analytic and simulation estimates of the expected cluster closing times for model 2. There is a loose relationship between the expected opening time, and the agreement between simulation and analytic results, such that if the expected opening time is long it is likely the agreement will be worse than if the expected opening time is short. Thus, it seems that the error results from the smallest eigenvalues of the coefficient matrix.

We tried a variety of criteria for the degree of state aggregation still providing good approximations. We required for instance the inverse of the smallest transition rate lumped to be much smaller than moments of the waiting time distributions or residence times of the system in aggregates. These are criteria suggested by the use of time scale separation as the basis for state aggregation. Surprisingly, meeting conditions of that type did not give conclusive predictions on the quality of the approximation. The criterium to stop lumping states into aggregates when analytic single channel results get bad compared to single channel simulations was the most predictive and feasible criterium.

We have not related our results to experimental observations in this study yet. Fitting models to experimental data will be the subject of future research. Puffs can be found in a very small range of $\mathrm{IP}_{3}$ concentration only below which no release is observed and above which spiking or sustained release is found. Only recently. the lab of I. Parker's developed a method to observe puffs over a larger range of $\mathrm{IP}_{3}$ concentrations by decoupling puff sites by the buffer EGTA [22]. This method will produce data with which we can meaningfully compare our results. 


\section{References}

[1] Adkins, C. E., and C. W. Taylor. 1999. Lateral Inhibition of inositol 1,4,5trisphosphate receptors by cytosolic $\mathrm{Ca}^{2+}$. Curr. Biol. 9:1115-1118.

[2] Callamaras, N., J. S. Marchant, X.-P. Sun, and I. Parker. 1998. Activation and co-ordination of $\mathrm{InsP}_{3}$-mediated elementary $\mathrm{Ca}^{2+}$ events during global $\mathrm{Ca}^{2+}$ signals in Xenopus oocytes. J. Physiol. 509:81-91.

[3] De Young, G. W., and J. Keizer. 1992. A Single-pool inositol 1,4,5-trisphosphatereceptor-based model for agonist-stimulated oscillations in $\mathrm{Ca}^{2+}$ concentration. Proc. Natl. Acad. Sci. USA. 89:9895-9899.

[4] Deuflhard, P., W. Huisinga, A. Fischer, Ch. Schütte. 2000. Identification of almost invariant aggregates in reversible nearly uncoupled Markov chains. Linear Algebra and its Applications. 315:39-59

[5] Dupont, G., and S. Swillens. 1995. Quantal release, incremental detection, and long-period $\mathrm{Ca}^{2+}$ oscillations in a model based on regulatory $\mathrm{Ca}^{2+}$-binding sites along the permeation pathway. Biophys. J. 71:1714-1722.

[6] Falcke, M. 2003. On the role of stochastic channel behavior in intracellular $\mathrm{Ca}^{2+}$ dynamics. Biophys. J. 84:42-56

[7] Falcke, M. 2004. Reading the patterns in living cells - the physics of $\mathrm{Ca}^{2+}$ signaling. Adv. Phys. 53:255-440.

[8] Fraiman, D., B. Pando, S. Dargan, I. Parker, and S. P. Dawson. 2006. Analysis of Puff Dynamics in Oocytes: Interdependence of Puff Amplitude and Interpuff Interval. Biophys. J. 90:3897-3907.

[9] Nguyen V., R. Mathias, G. Smith. 2005. A stochastic automata network descriptor for markov chain models of instantaneously-coupled intracellular $\mathrm{Ca}^{2+}$ channels. Bull. Math. Biol. 67:393-432 
[10] Rüdiger, S., J. W. Shuai, W. Huisinga, C. Nagaiah, G. Warnecke, I. Parker, M. Falcke. 2007. Hybrid Stochastic and Deterministic Simulations of Calcium Blips. Biophys. J. 93:1847-1857.

[11] Shuai, J.W., P. Jung. 2002. Stochastic properties of $\mathrm{Ca}^{2+}$ release of inositol 1,4,5trisphosphate receptor clusters. Biophys.J. 83:87-97

[12] Shuai, J., J. E. Pearson, J. K. Foskett, D. D. Mak and, I. Parker. 2007. A Kinetic Model of Single Clustered $\mathrm{IP}_{3}$ Receptors in the Absence of $\mathrm{Ca}^{2+}$ Feedback. Biophys. J. 93:1151-1162.

[13] Skupin, A., H. Kettenmann, U. Winkler, M. Wartenberg, H. Sauer, St. C. Tovey, C. W. Taylor, M. Falcke. 2008. How does intracellular $\mathrm{Ca}^{2+}$ oscillate: by chance or by the clock?. Biophys. J. 94:

[14] Sneyd, J., and J. F. Dufour. 2002. A dynamic model of the type-2 inositol trisphosphate receptor. Proc. Natl. Acad. Sci. USA. 99:2398-2403.

[15] Sneyd, J., and M. Falcke. 2005. Models of the inositol trisphosphate receptor. Prog. Biophys. Mol. Biol. 89:207-245.

[16] Sun, X.-P., N. Callamaras, J. S. Marchant, and I. Parker. 1998. A continuum of $\mathrm{InsP}_{3}$-mediated elementary $\mathrm{Ca}^{2+}$ signalling events in Xenopus oocytes. J. Physiol. 509:67-80.

[17] Swillens, S., G. Dupont, L. Combettes, and P. Champell. 1999. From calcium blips to calcium puffs: theoretical analysis of the requirements for interchannel communication. Proc. Natl. Acad. Sci. USA. 96:13750-13755.

[18] Thul, R., and M. Falcke. 2007. Waiting time distributions for clusters of complex molecules. Europhysics Letters 79:38003.

[19] Ullah, G., and P. Jung. 2006. Modeling the Statistics of Elementary Calcium Release Events. Biophys. J. 90:3485-3495. 
[20] Demuro, A. and I. Parker. 2008. Multi-dimensional resolution of elementary Ca ${ }^{2+}$ signals by simultaneous multi-focal imaging, Cell Calcium 43(4):367-374

[21] Taufiq-Ur-Rahman, A. Skupin, M. Falcke, C.W. Taylor. 2009. Clustering of $\mathrm{IP}_{3}$ receptors by $\mathrm{IP}_{3}$ retunes their regulation by $\mathrm{IP}_{3}$ and $\mathrm{Ca}^{2+}$, Nature, in press

[22] Smith, F., S.M. Wiltgen, and I. Parker. 2009. Localization of puff sites adjacent to the plasma membrane: Functional and spatial characterization of $\mathrm{Ca}^{2+}$ signaling in SH-SY5Y cells utilizing membrane-permeant caged $\mathrm{IP}_{3}$, Cell Calcium 45:65-76 
Table 1: Parameter values for Model 1

\begin{tabular}{llll}
\hline Parameter & Value & Parameter & Value \\
\hline$a_{0}$ & $550.0 s^{-1}$ & $b_{0}$ & $80.0 s^{-1}$ \\
$K_{1}$ & $0.0036 \mu \mathrm{M}$ & $a_{1}$ & $60.0 \mu \mathrm{M}^{-1} s^{-1}$ \\
$K_{2}$ & $16.0 \mu \mathrm{M}$ & $a_{2}$ & $0.2 \mu \mathrm{M}^{-1} s^{-1}$ \\
$K_{3}$ & $0.8 \mu \mathrm{M}$ & $a_{3}$ & $5.0 \mu \mathrm{M}^{-1} s^{-1}$ \\
$K_{4}$ & $0.072 \mu \mathrm{M}$ & $a_{4}$ & $0.5 \mu \mathrm{M}^{-1} s^{-1}$ \\
$K_{5}$ & $0.8 \mu \mathrm{M}$ & $a_{5}$ & $150.0 \mu \mathrm{M}^{-1} s^{-1}$ \\
\hline
\end{tabular}


Table 2: Parameter values for Model 3

\begin{tabular}{llll}
\hline Parameter & Value & Parameter & Value \\
\hline$k_{1}$ & $2.0 \mu \mathrm{M}^{-1} s^{-1}$ & $k_{-1}$ & $0.04 s^{-1}$ \\
$k_{2}$ & $37.4 \mu \mathrm{M}^{-1} s^{-1}$ & $k_{-2}$ & $1.4 s^{-1}$ \\
$k_{3}$ & $0.11 s^{-1}$ & $k_{-3}$ & $29.8 s^{-1}$ \\
$k_{4}$ & $4.0 \mu \mathrm{M}^{-1} s^{-1}$ & $k_{-4}$ & $0.37 s^{-1}$ \\
$k_{5}$ & $2.0 \mu \mathrm{M}^{-1} s^{-1}$ & $l_{1}$ & $10.0 \mu \mathrm{M}^{-1} s^{-1}$ \\
$l_{3}$ & $100.0 \mu \mathrm{M}^{-1} s^{-1}$ & $l_{5}$ & $0.1 \mu \mathrm{M}^{-1} s^{-1}$ \\
$L_{1}$ & $0.12 \mu \mathrm{M}^{2}$ & $L_{3}$ & $0.025 \mu \mathrm{M}$ \\
$L_{5}$ & $38.2 \mu \mathrm{M}$ & $l_{2}$ & $1.7 s^{-1}$ \\
$l_{-2}$ & $0.8 s^{-1}$ & $l_{4}$ & $37.4 \mu \mathrm{M}^{-1} s^{-1}$ \\
$l_{-4}$ & $2.5 s^{-1}$ & $l_{6}$ & $4707.0 s^{-1}$ \\
$l_{-6}$ & $11.4 s^{-1}$ & & \\
\hline
\end{tabular}


Figure 1: The nine state model of an $\mathrm{IP}_{3}$ receptor channel subunit proposed by Rüdiger et al. [10]. The $\mathrm{Ca}^{2+}$ concentration is denoted by $c$ and the $\mathrm{IP}_{3}$ concentration is denoted by $p$. The dotted lines indicate the transitions that are made small in model 2.

Figure 2: The model of an $\mathrm{IP}_{3}$ receptor channel subunit given by Sneyed [14]. The channel states are $R$ : unbound receptor, $I_{1}$ and $I_{2}$ : inactivated, $O$ : open, $A$ : activated, and $S$ : shut. The $\mathrm{Ca}^{2+}$ concentration is denoted by $c$ and the $\mathrm{IP}_{3}$ concentration is denoted by $p$.

Figure 3: Open probability distribution $F_{\text {open }}(A, t)$ for various $c_{\text {close }}$ values. A: $F_{\text {open }}(A, t)$ for model 1. B: $F_{\text {open }}(A, t)$ for model 2. C: $F_{\text {open }}(A, t)$ for model 3.

Figure 4: A: The expected opening times $T_{\text {open }}$ for the three models. B: Enlarged plot of the expected opening times for the three models to show clearly the minimum of models 1 and 2 for small values of $c_{\text {open }}$.

Figure 5: Opening time distribution $F_{\text {open }}(A, t)$ when changing $p$ for some representative $\mathrm{IP}_{3}$ concentrations. A: $F_{\text {open }}(A, t)$ for model 1. B: $F_{\text {open }}(A, t)$ for model 2. $\mathrm{C}: F_{\text {open }}(A, t)$ for model 3.

Figure 6: A: The expected opening times $T_{\text {open }}$ for the three models as function of the $\mathrm{IP}_{3}$ concentration. B: Enlarged plot of $T_{\text {open }}$ for small concentrations.

Figure 7: Cluster closing time distribution $F_{\text {close }}(A, t)$ when changing $c_{\text {open }}$. A: $F_{\text {close }}(A, t)$ for model 1. B: $F_{\text {close }}(A, t)$ for model 2. C: $F_{\text {close }}(A, t)$ for model 3.

Figure 8: The expected closing times $T_{\text {close }}$ for the three models. Model 2 is represented by the upper lines, where the dashed line with $\times$-symbols show the simulation results and dashed line without shows analytic results. Model 1 is shown by two 
full lines, with +-symbols for simulation and without for analytic results. Fast $\mathrm{IP}_{3}$ release in model 2 corresponds to $K_{1}=0.036 \mu \mathrm{M}$ and $K_{3}=8.0 \mu \mathrm{M}$. The simulation results are represented by the dashed line with $\square$-symbols and the analytic results by the dashed line. Results for model 3, analytic and simulation, is shown by the dashed line with the $*$-symbols below. The analytic and simulation results can not be distinguished.

Figure 9: Cluster closing time distribution $F_{\text {close }}(A, t)$ when changing $p$. A: $F_{\text {close }}(A, t)$ for model 1. B: $F_{\text {close }}(A, t)$ for model 2. C: $F_{\text {close }}(A, t)$ for model 3.

Figure 10: A: The expected closing times $T_{\text {close }}$ for models 1 and 2 , from both the analytic and simulation results. Model 1 is represented by the upper dashed line (analytic results) and dashed line with $\times$-symbols (simulation results). Model 2 is represented by the lower full lines. There are few differences between analytic (full line) and simulation results (full line with +-symbols). B: The expected closing times $T_{\text {close }}$ for model 3 only with analytic (full line) and simulation results (full line with + -symbols).

Figure 11: $F_{\text {close }}(A, t)$ using both a fixed and variable $\mathrm{Ca}^{2+}$ concentration. Here $c_{\text {close }}=0.1 \mu \mathrm{M}$ and $p=0.15 \mu \mathrm{M}$. $c_{\text {open }}=100 \mu \mathrm{M}$ when fixed. When $c_{\text {open }}$ is variable, it has a value of $100 \mu \mathrm{M}$ for a channel which is open and a value of $10.09 \mu \mathrm{M}$ (labeled 'Variable $c_{\text {open }} 1^{\prime}$ ') or $1.099 \mu \mathrm{M}$ (labeled 'Variable $c_{\mathrm{open}} 2$ ') for a channel which is closed. A: Results for model 1. B: Results for model 2. C: Results for model 3.

Figure 12: Analytic and simulation results showing $F_{\text {open }}(A, t)$ for all three models. Here $c_{\text {close }}=0.1 \mu \mathrm{M}$, and $p=0.15 \mu \mathrm{M}$. The full lines represent the analytic results and the $\square$-symbols the simulation results. A: Results for model 1. B: Results for model 2. C: Results for model 3.

Figure 13: Analytic and simulation results showing $F_{\text {close }}(A, t)$ for the three mod- 
els. Here, $c_{\text {open }}=100 \mu \mathrm{M}$ and $p=0.15 \mu \mathrm{M}$. A: $F_{\text {close }}(A, t)$ for model 1. B: $F_{\text {close }}(A, t)$ for model 2. C: $F_{\text {close }}(A, t)$ for model 3.

Figure 14: The effect of the number of aggregate states on the expected closing time. These results are for a single channel. A-C show the expected closing time $T_{\text {close }}$ as the number of aggregate states is reduced. This is shown for the three models at various parameter values. The vertical lines represent the number of aggregate states which are used in the results presented here. D-F show the number of open aggregates $N_{\text {open }}$ as function of the aggregate states for all three models and various parameters.

Figure 15: Varying the cluster size. Graphs A, B and C show the expected closing time of the cluster $T_{\text {close }}$, using models $1(\mathrm{~A}), 2(\mathrm{~B})$ and $3(\mathrm{C})$ respectively, as the number of channels in the cluster is varied from 1 to 5 . We use $p=0.15 \mu \mathrm{M}$, $c_{\text {open }}=100 \mu \mathrm{M}$ and $c_{\text {close }}=0.1 \mu \mathrm{M}$ (to compute the initial condition). Both analytic (full lines) and simulation results (dashed lines with +-symbols) are shown. 


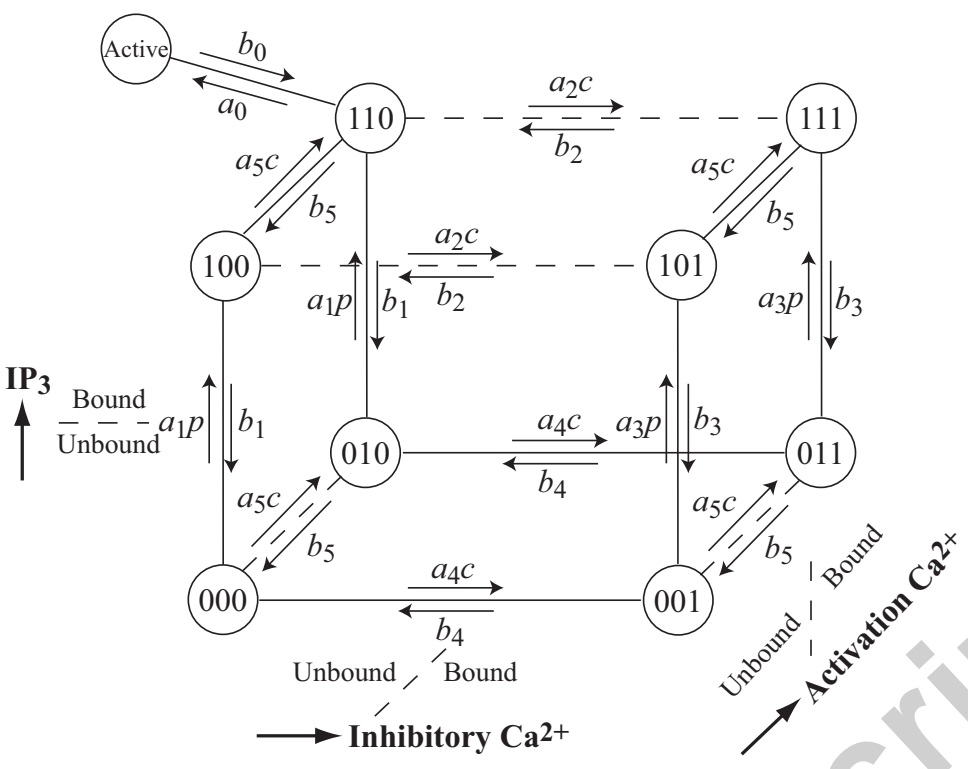

Figure 1: 

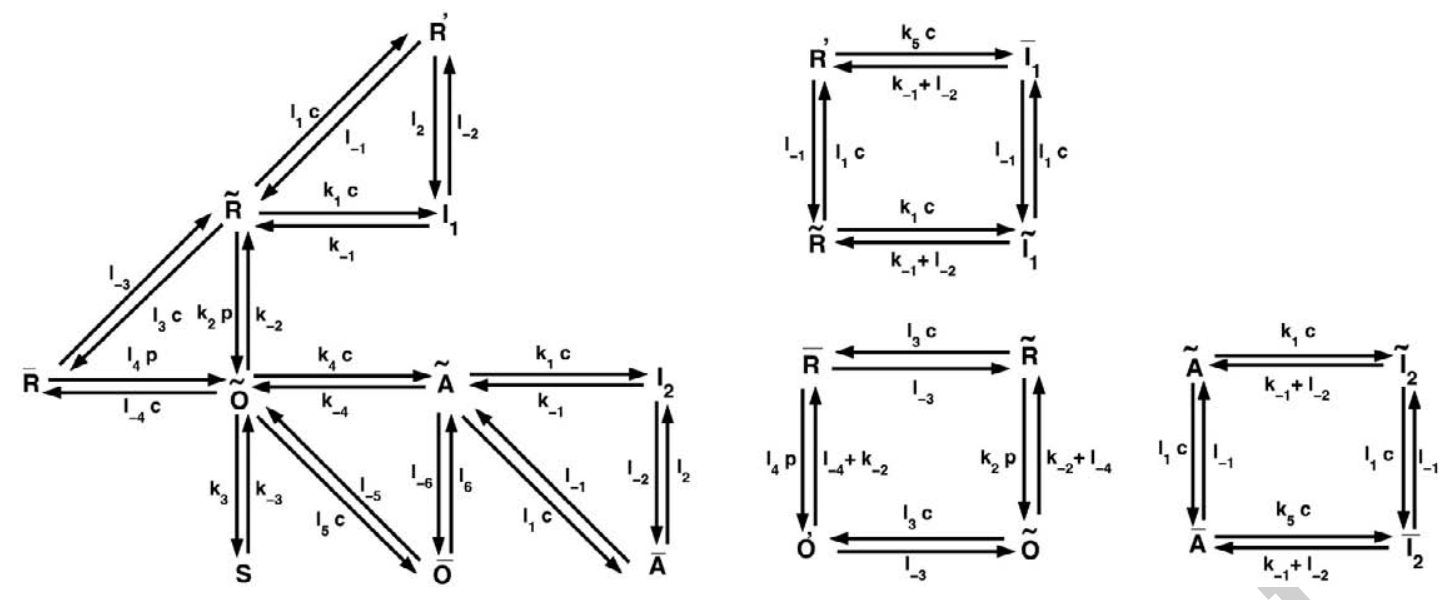

Figure 2: 

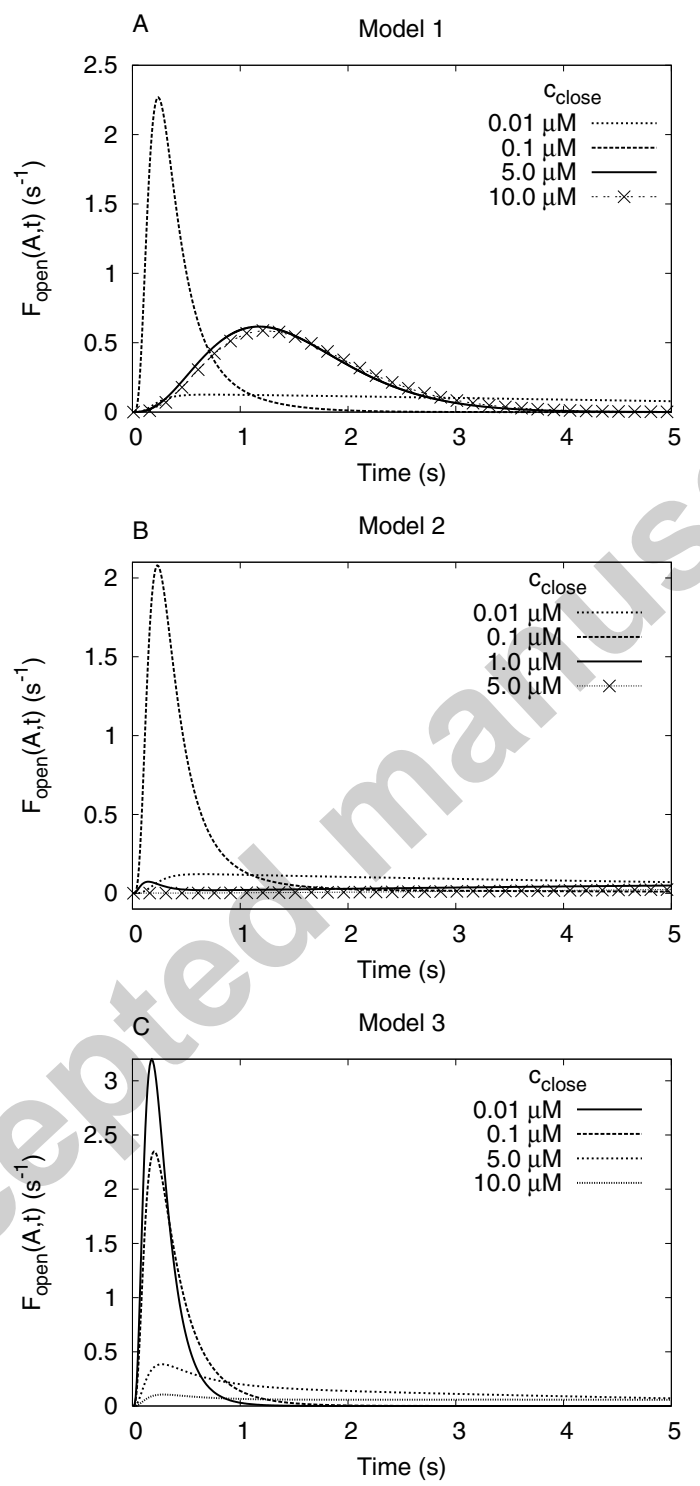

Figure 3: 

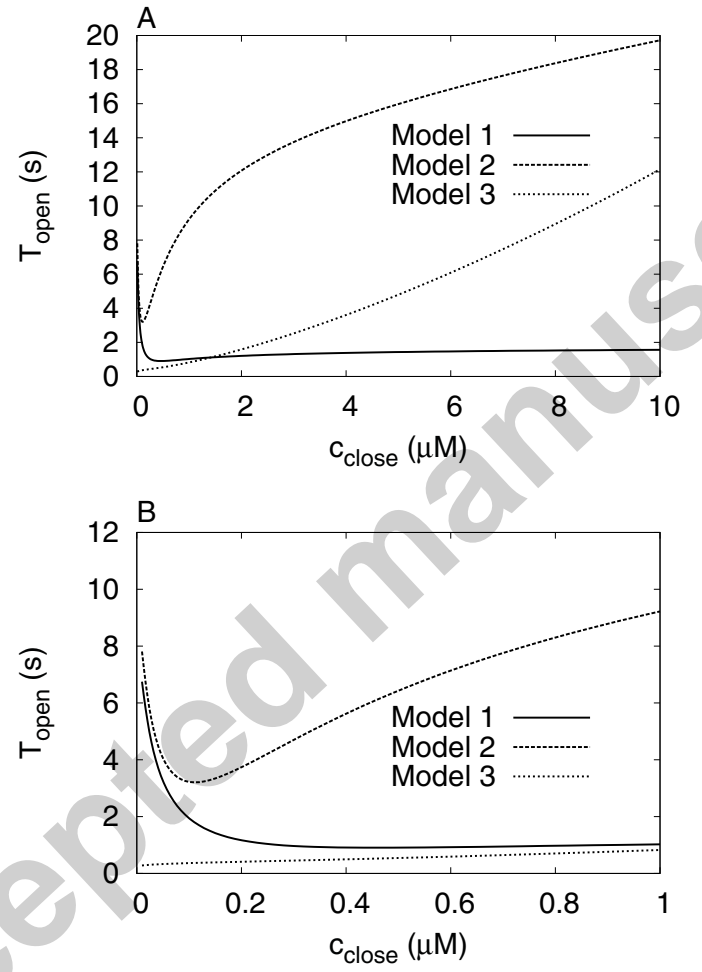

Figure 4: 

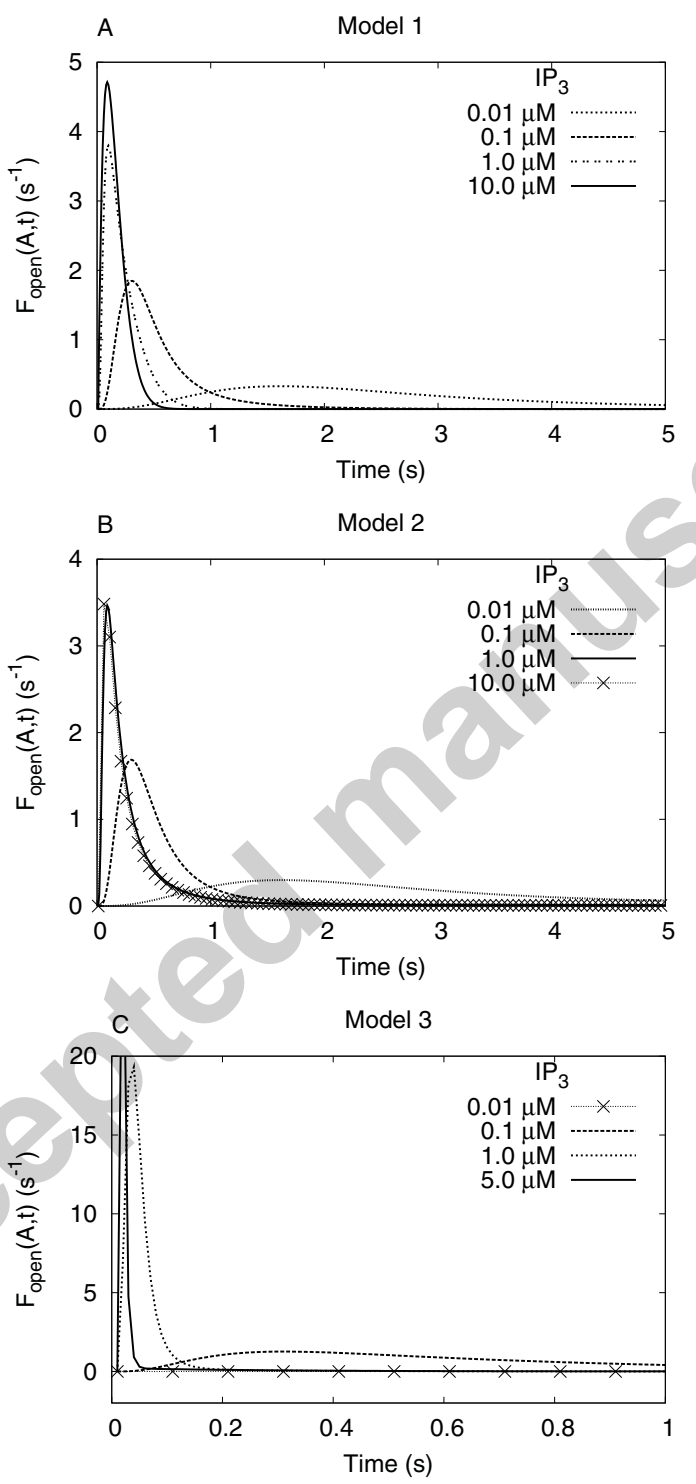

Figure 5: 

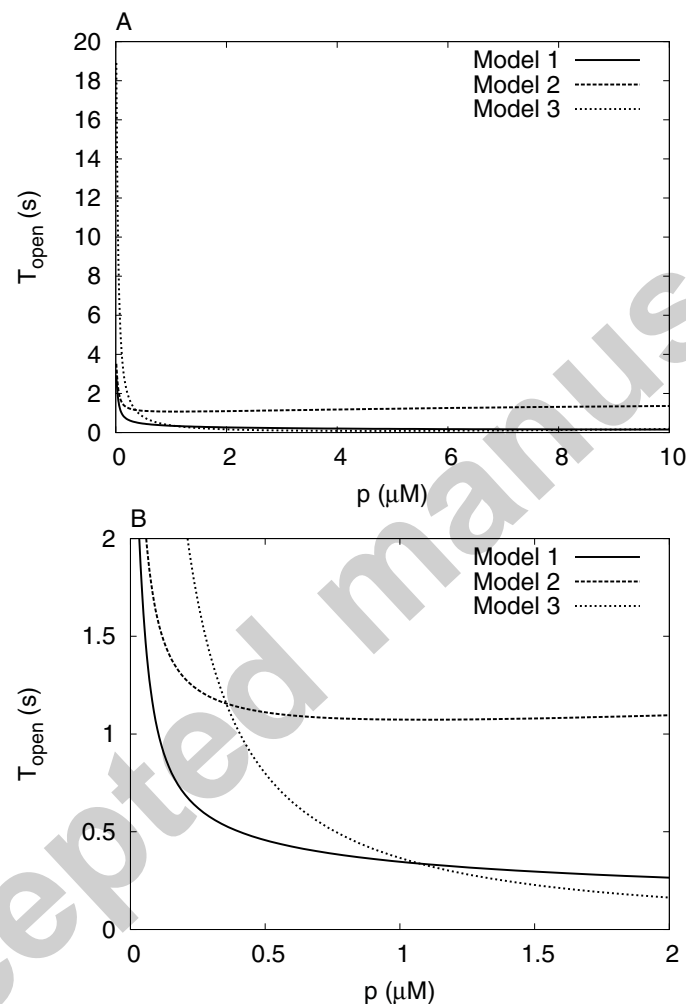

Figure 6: 

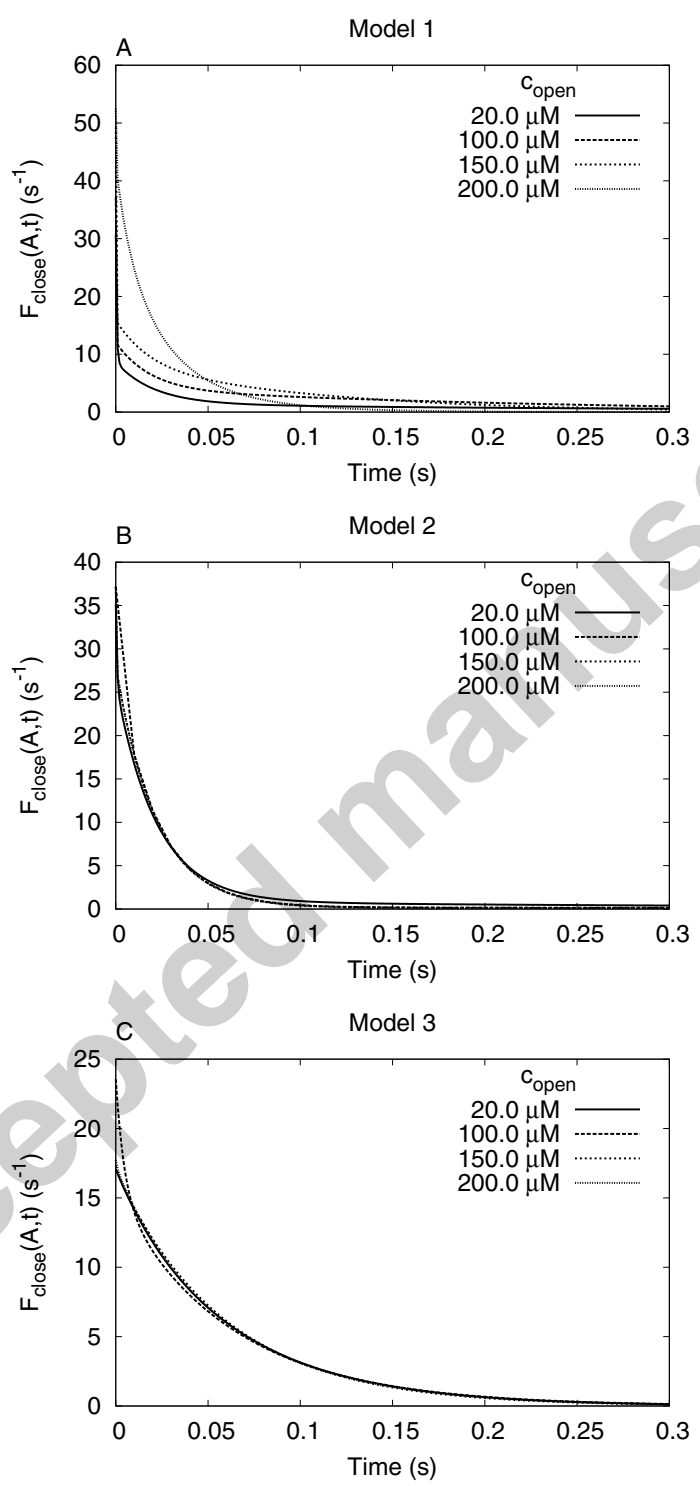

Figure 7: 


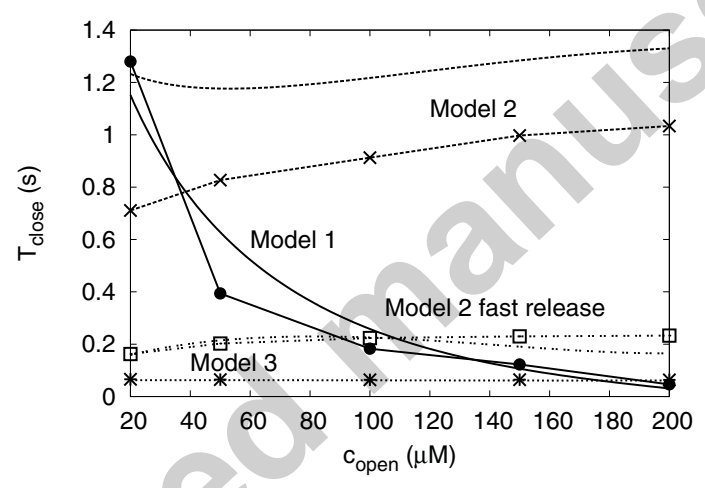

Figure 8: 

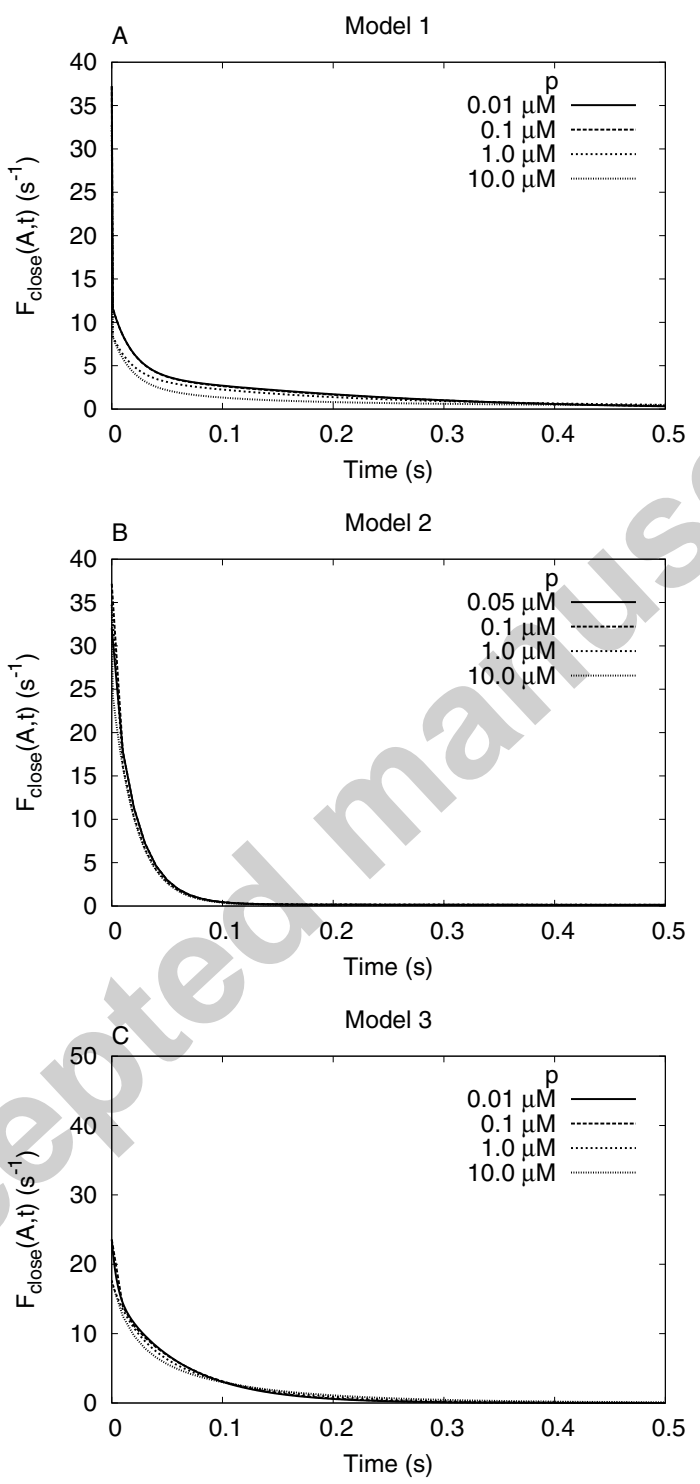

Figure 9: 

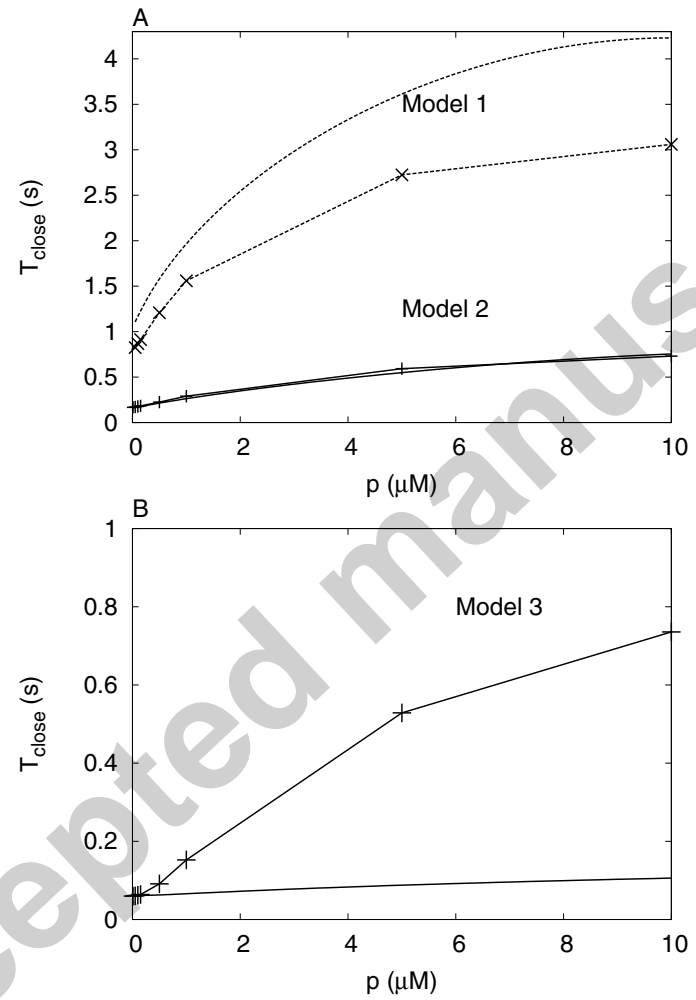

Figure 10: 

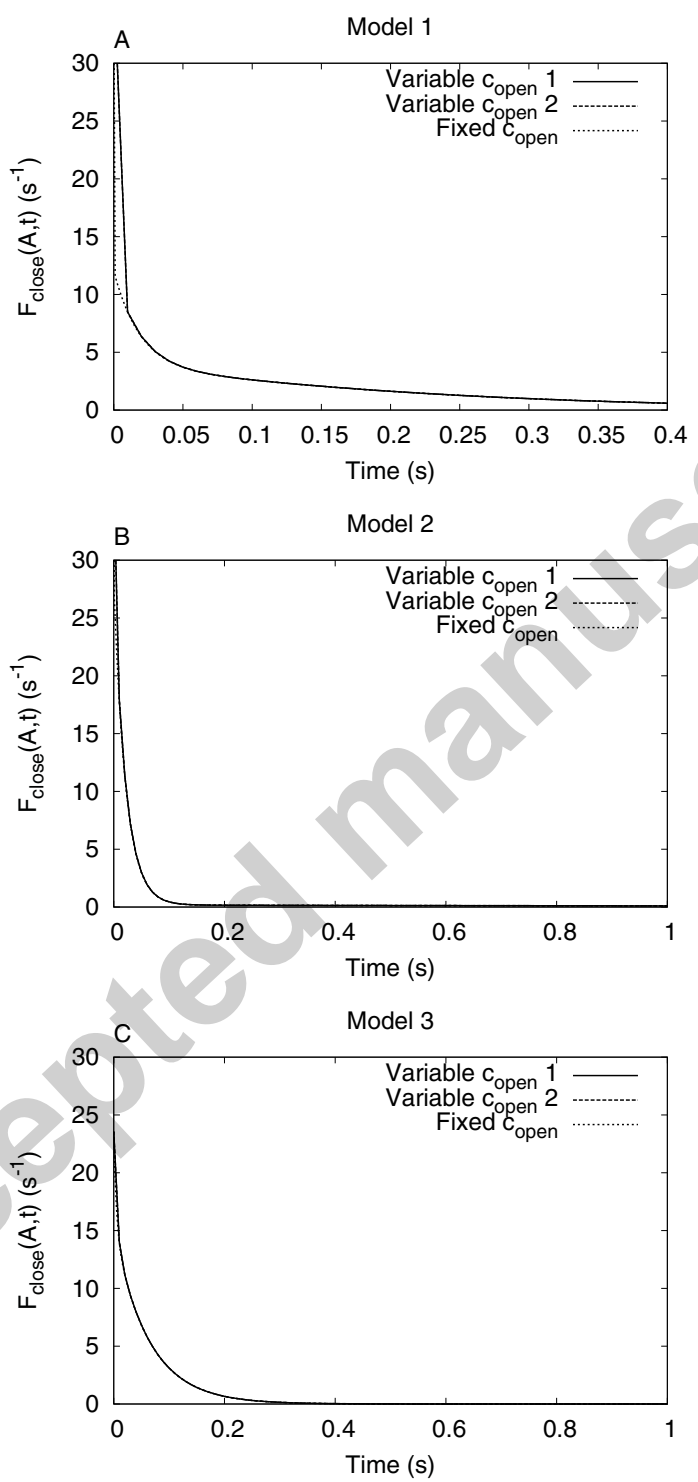

Figure 11: 

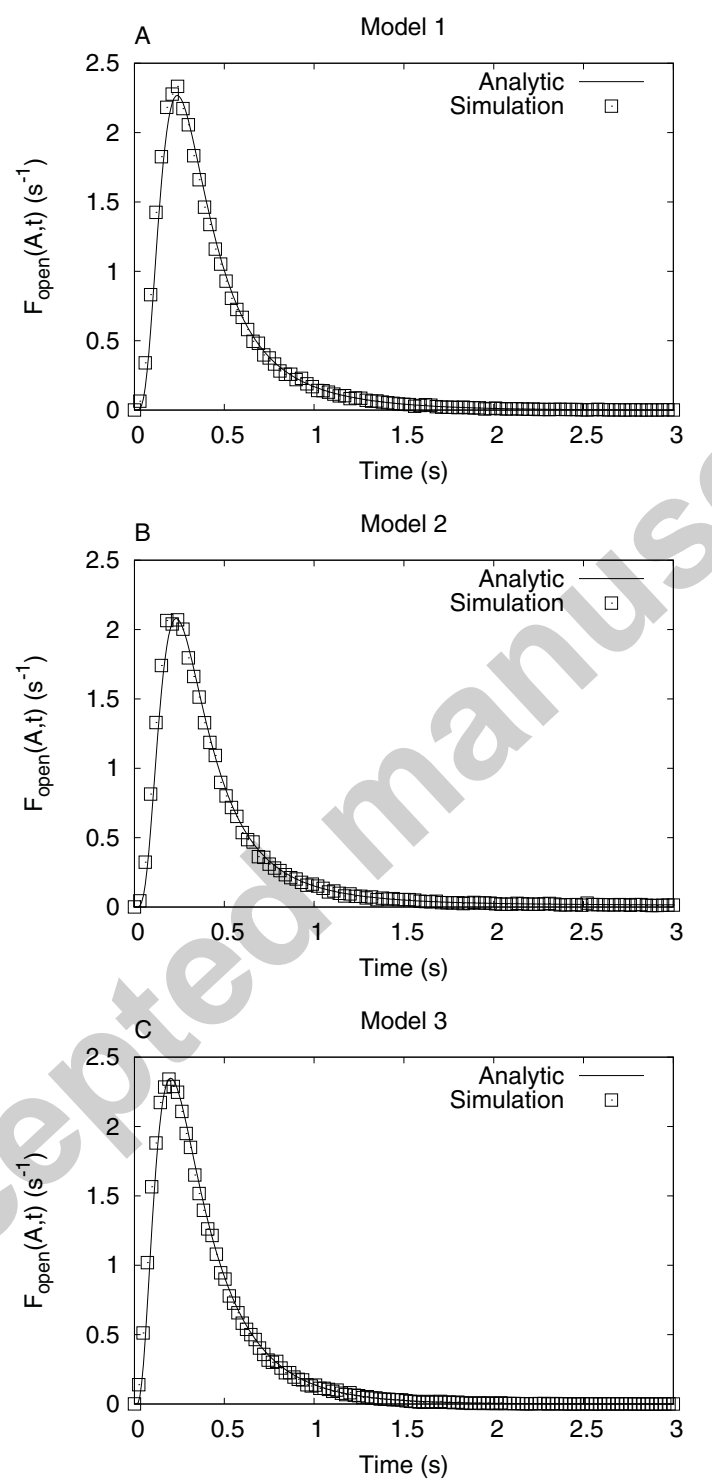

Figure 12: 

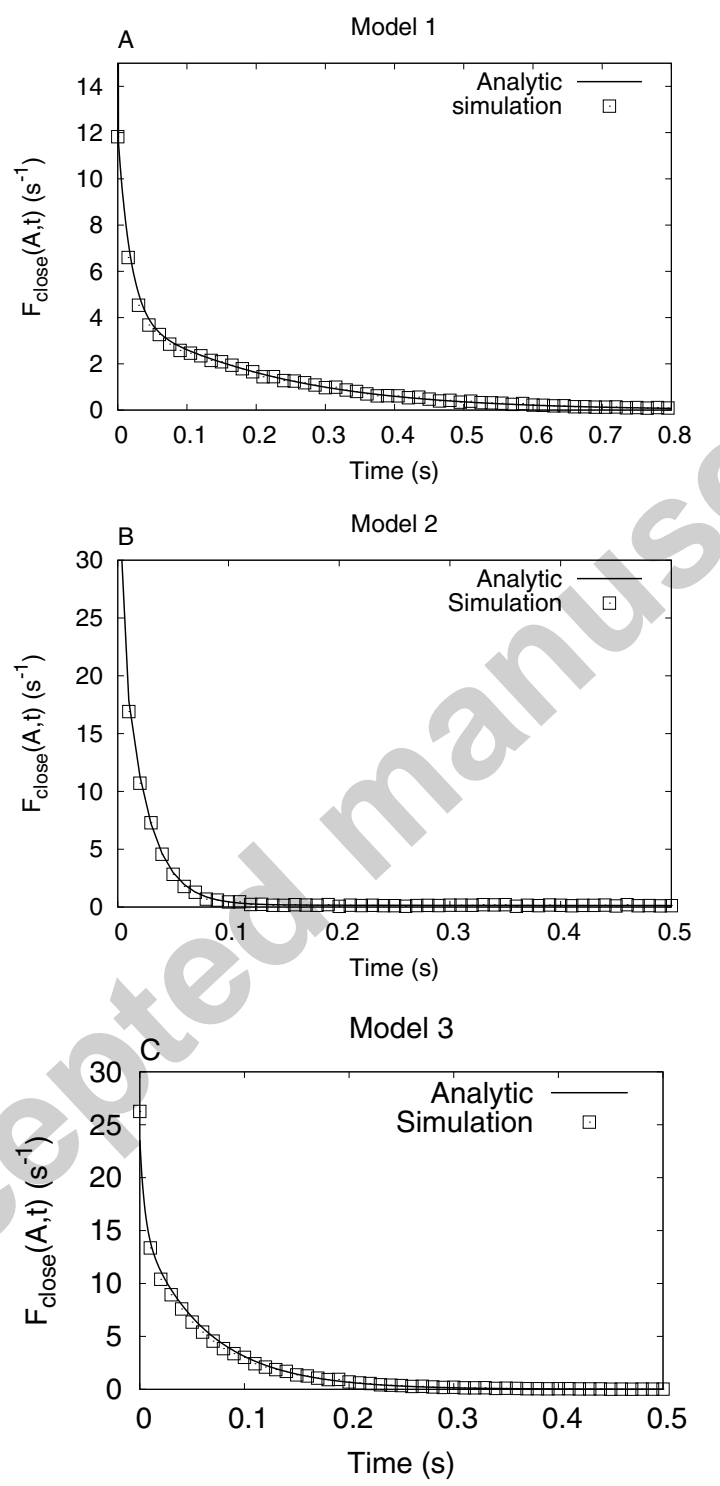

Figure 13: 

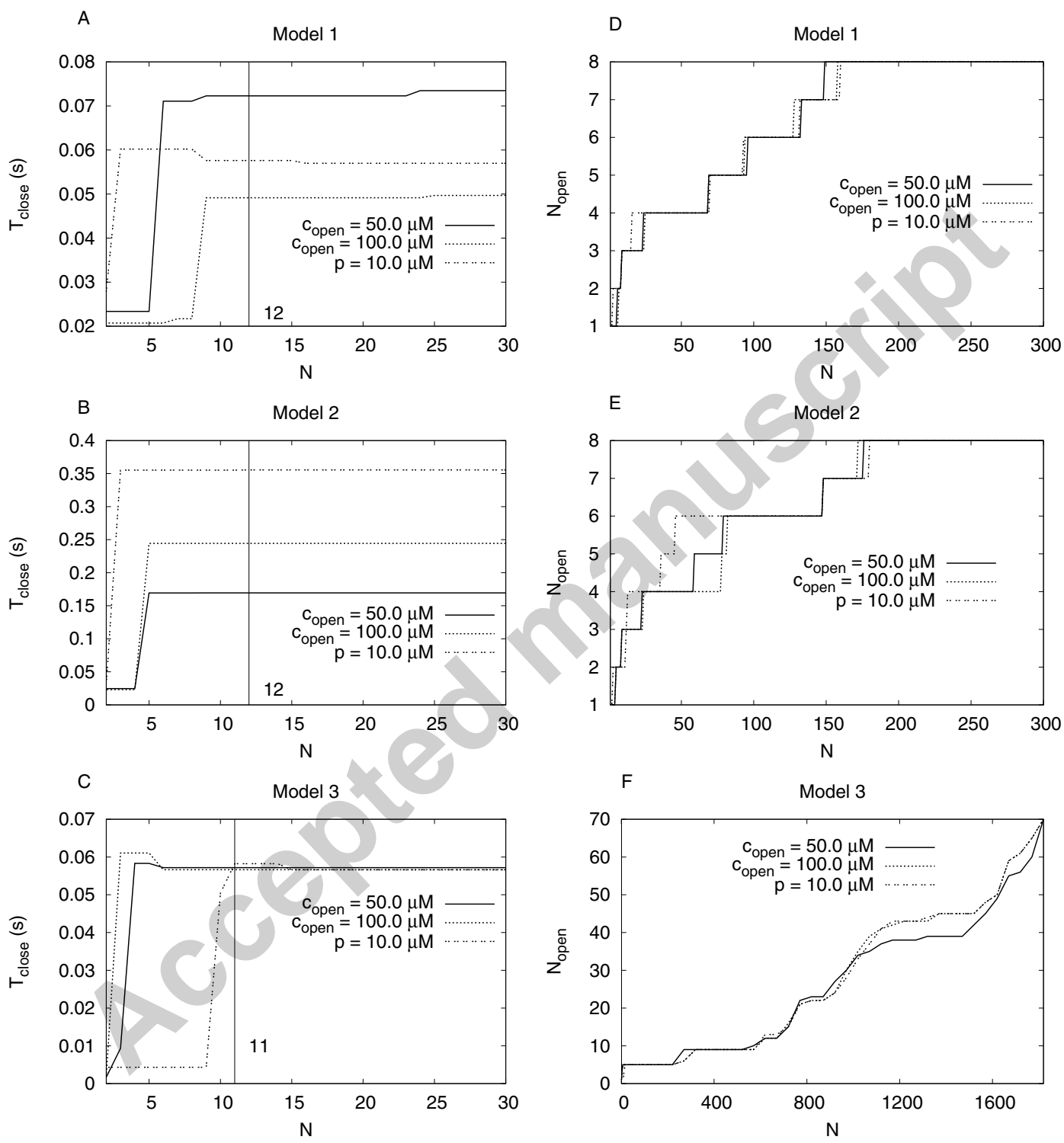

Figure 14: 

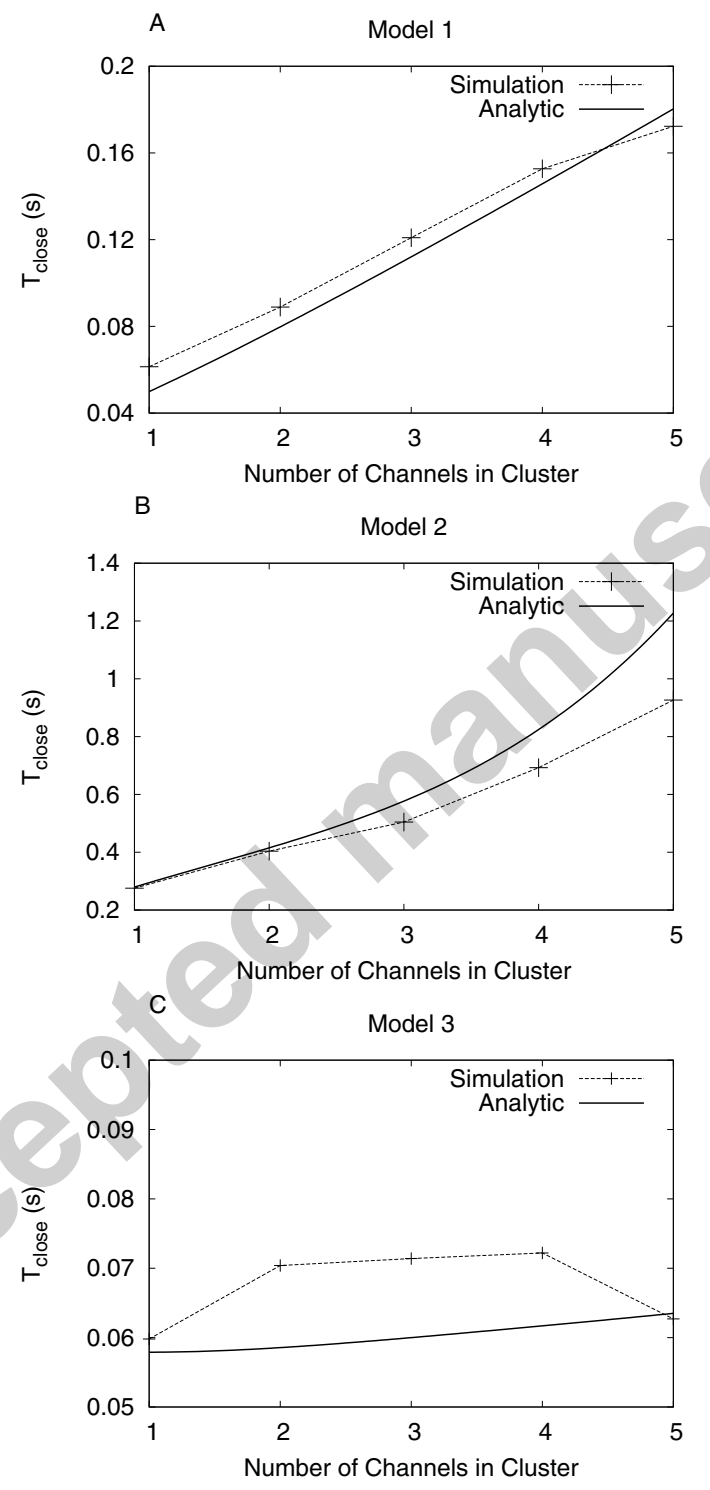

Figure 15: 